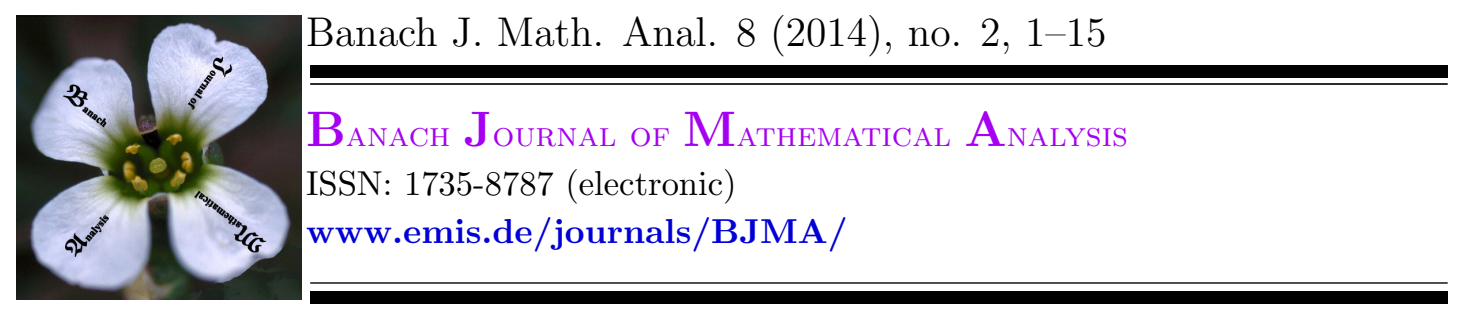

\title{
COMPACT OPERATORS IN THE COMMUTANT OF ESSENTIALLY NORMAL OPERATORS
}

\author{
H. S. MUSTAFAYEV ${ }^{1 *}$ AND F. B. HÜSEYNOV ${ }^{2}$ \\ Communicated by D. Bakić
}

\begin{abstract}
Let $T$ be a bounded, linear operator on a complex, separable, infinite dimensional Hilbert space $H$. We assume that $T$ is an essentially isometric (resp. normal) operator, that is, $I_{H}-T^{*} T$ (resp. $T T^{*}-T^{*} T$ ) is compact. For the compactness of $S$ from the commutant of $T$, some necessary and sufficient conditions are found on $S$. Some related problems are also discussed.
\end{abstract}

\section{INTRODUCTION AND PRELIMINARIES}

Let $H$ be a complex, separable, infinite dimensional Hilbert space and let $B(H)$ be the algebra of all bounded, linear operators on $H$. As usual, we denote the spectrum (resp. left, right) of $T \in B(H)$ by $\sigma(T)$ (resp. $\sigma_{l}(T), \sigma_{r}(T)$ ). The unit circle in the complex plane will be denoted by $\Gamma$, whereas $D$ indicates the open unit disk. The disc-algebra and the algebra of all bounded analytic functions on $D$ are denoted by $A(D)$ and $H^{\infty}:=H^{\infty}(D)$, respectively.

If $T \in B(H)$, we let $A_{T}$ denote the closure in the uniform operator topology of all polynomials in $T$. Notice that $A_{T}$ is a commutative unital Banach algebra. The Gelfand space of $A_{T}$ can be identified with $\sigma_{A_{T}}(T)$, the spectrum of $T$ with respect to the algebra $A_{T}$. Since $\sigma(T)$ is a (closed) subset of $\sigma_{A_{T}}(T)$, for every $\lambda \in \sigma(T)$ there exists a multiplicative functional $\phi_{\lambda}$ on $A_{T}$ such that $\phi_{\lambda}(T)=\lambda$.

By $\widehat{S}$, we will denote the Gelfand transform of $S \in A_{T}$. Here and in the sequel,

Date: Received: Feb. 11, 2013; Revised: May 21, 2013; Accepted: Aug. 7, 2013.

* Corresponding author.

2010 Mathematics Subject Classification. Primary 47A10; Secondary 47A53, 47A60, 47B07.

Key words and phrases. Compact operator, essentially unitary (normal) operator, (essential) spectrum, functional calculus. 
instead of $\widehat{S}\left(\phi_{\lambda}\right)\left(=\phi_{\lambda}(S)\right)$, where $\lambda \in \sigma(T)$, we will use the notation $\widehat{S}(\lambda)$. Notice that $\lambda \mapsto \widehat{S}(\lambda)$ is a continuous function on $\sigma(T)$.

Recall that $\sigma(T) \cap \Gamma$ is called the unitary spectrum of $T \in B(H)$. It follows from the Shilov's Theorem [7, Theorem 2.3.1] that if $T$ is a contraction, then

$$
\sigma_{A_{T}}(T) \cap \Gamma=\sigma(T) \cap \Gamma
$$

A contraction $T$ on $H$ is said to be completely nonunitary (c.n.u.) if it has no proper reducing subspace on which it acts as a unitary operator. If $T$ is a c.n.u. contraction, then $f(T)\left(f \in H^{\infty}\right)$ can be defined by the Nagy-Foias functional calculus [13, Chapter III]. We put $H^{\infty}(T):=\left\{f(T): f \in H^{\infty}\right\}$. A c.n.u. contraction $T$ is called a $C_{0}$-contraction if there exists a nonzero function $f \in H^{\infty}$ such that $f(T)=0$. B. Sz.-Nagy [12] proved that if $T$ is a $C_{0}$-contraction, then the commutant $\{T\}^{\prime}:=\{S \in B(H): T S=S T\}$ of $T$ contains a nonzero compact operator, but there exists a $C_{0}$-contraction $T$ such that zero is the unique compact operator contained in $H^{\infty}(T)$. An operator $T \in B(H)$ is said to be essentially unitary if both $I_{H}-T^{*} T$ and $I_{H}-T T^{*}$ are compact. Nordgren [16] proved that if $T$ is an essentially unitary $C_{0}$-contraction, then $H^{\infty}(T)$ contains a nonzero compact operator.

If $T$ is a contraction on $H$, then it follows from the von Neumann inequality that there exists a contractive algebra homomorphism $h: A(D) \rightarrow A_{T}$ (with dense range) such that $h(1)=I_{H}$ and $h(z)=T$. We will use the notation $f(T):=h(f), f \in A(D)$. Thus we have $\|f(T)\| \leq\|f\|_{\infty}$ for all $f \in A(D)$.

Recall that $T \in B(H)$ is called essentially isometric operator if $I_{H}-T^{*} T$ is compact. Kellay and Zarrabi [6] proved that if the essentially isometric contraction $T$ satisfies the condition $D \backslash \sigma(T) \neq \emptyset$ (it follows that $T$ is a compact perturbation of a unitary operator and therefore it is essentially unitary) and if $f \in A(D)$ vanishes on $\sigma(T) \cap \Gamma$, then $f(T)$ is compact. Notice that under the above conditions the Lebesgue measure of $\sigma(T) \cap \Gamma$ is necessarily zero. In [6], it is also shown that if $T$ is an essentially isometric $C_{0}$-contraction, then $f(T)$ $\left(f \in H^{\infty}\right)$ is compact if and only if $\lim _{n \rightarrow \infty}\left\|T^{n} f(T)\right\|=0$. The proofs of these results essentially use the Beurling-Rudin theorem about the structure of closed ideals of $A(D)$ and the corona theorem.

By $K(H)$ we will denote the ideal of compact operators on $H$. The quotient algebra $B(H) / K(H)$ is a $C^{*}$-algebra called the Calkin algebra. Let $\pi: B(H) \rightarrow B(H) / K(H)$ be the canonical map. The essential spectrum $\sigma_{e}(T)$ of $T \in B(H)$ is the spectrum of $\pi(T)$ in the Calkin algebra. As is well known, $\sigma_{e}(T)$ is a nonempty compact subset of $\sigma(T)$. Similarly, the left and right essential spectrum of $T$ are defined by $\sigma_{l e}(T):=\sigma_{l}(\pi(T))$ and $\sigma_{r e}(T):=\sigma_{r}(\pi(T))$. Recall also that $T \in B(H)$ is a (left, right) Fredholm operator if $\pi(T)$ is (left, right) invertible in the Calkin algebra.

The main results of this note can be summarized as follows. If $T$ is a c.n.u. contraction and $S \in A_{T}$ is compact, then $\widehat{S}$ vanishes on $\sigma(T) \cap \Gamma$. If $T$ is an essentially isometric operator and if the Gelfand transform of $S \in A_{T}$ vanishes on $\sigma_{l e}(T)$ (or on $\sigma_{r e}(T) \cap \Gamma$ ), then $S$ is compact. In addition if $T$ is a c.n.u. 
contraction, then $S \in\{T\}^{\prime}$ is compact if and only if

$$
\lim _{n \rightarrow \infty}\left\|T^{n} S\right\|=0 \text {. }
$$

Furthermore, the compactness of $S \in\{T\}^{\prime}$ characterized via the ergodic conditions. If

$$
\varliminf_{n \rightarrow \infty} \frac{1}{n}\left\|\sum_{k=1}^{n} \bar{\xi}^{k} T^{k} S\right\|=0
$$

holds for every $\xi \in \sigma_{l e}(T)$ (or $\xi \in \sigma_{r e}(T) \cap \Gamma$ ), then $S$ is compact.

Similar results for essentially normal operators are also obtained. Let $T$ be an essentially normal operator. If the Gelfand transform of $S \in A_{T}$ vanishes on $\sigma_{e}(T)$, then $S$ is compact. In addition if $T$ is a Fredholm operator and if

$$
\varliminf_{n \rightarrow \infty} \frac{1}{n}\left\|\sum_{k=1}^{n} \lambda^{-k} T^{k} S\right\|=0
$$

holds for every $\lambda \in \sigma_{e}(T)$, then $S \in\{T\}^{\prime}$ is compact.

\section{Essentially isOMETRIC OPERATORS}

Let $T$ be an essentially isometric operator, that is, $I_{H}-T^{*} T$ is compact. In this section, for the compactness of the operator $S$ from the commutant of $T$, we give some necessary and sufficient conditions on $S$.

We start with the following result.

Proposition 2.1. Let $T$ be a c.n.u. contraction on $H$ and let $S \in A_{T}$. If $S$ is compact, then its Gelfand transform vanishes on $\sigma(T) \cap \Gamma$.

Proof. We know [9, Lemma 3.3] that if $T$ is a c.n.u. contraction, then $T^{n} \rightarrow 0$ in the weak operator topology. If $S \in A_{T}$ is compact, then for arbitrary $x \in H$ we can write

$$
\lim _{n \rightarrow \infty}\left\|T^{n} S x\right\|=\lim _{n \rightarrow \infty}\left\|S T^{n} x\right\|=0 .
$$

Since the set $\{S x:\|x\| \leq 1\}$ is relatively compact, for a given $\varepsilon>0$ it has a finite $\varepsilon$-mesh, say $\left\{S x_{1}, \cdots, S x_{k}\right\}$, where $\left\|x_{i}\right\| \leq 1(i=1, \cdots, k)$. Consequently, we have

$$
\left\|T^{n} S\right\| \leq \max _{i}\left\{\left\|T^{n} S x_{i}\right\|\right\}+\varepsilon(n \in \mathbb{N})
$$

It follows that $\lim _{n \rightarrow \infty}\left\|T^{n} S\right\|=0$. On the other hand, for every $\xi \in \sigma(T) \cap \Gamma$ there exists a multiplicative functional $\phi_{\xi}$ on $A_{T}$ such that $\phi_{\xi}(T)=\xi$. Since $\phi_{\xi}$ has norm one, we have

$$
|\widehat{S}(\xi)|=\left|\phi_{\xi}\left(T^{n} S\right)\right| \leq\left\|T^{n} S\right\| \rightarrow 0(n \rightarrow \infty) .
$$

Next, we have the following

Theorem 2.2. Let $T$ be an essentially isometric operator. If the Gelfand transform of $S \in A_{T}$ vanishes on $\sigma_{l e}(T)$ (or on $\sigma_{r e}(T) \cap \Gamma$ ), then $S$ is compact. 
For the proof we need some preliminary results.

Let $A$ be a $C^{*}$-algebra with the unit element $e$ and let $S_{A}$ be the set of all pure states on $A$. We know [14, Corollary V.23.3] that if $a \in A$, then $\sigma_{l}(a)$ consists of all $\lambda \in \mathbb{C}$ for which there exists $f \in S_{A}$ such that $\lambda=f(a)$ and $f\left(a^{*} a\right)=f\left(a^{*}\right) f(a)$. Assume that $a^{*} a=e$. If $\lambda \in \sigma_{l}(a)$, then we have

$$
|\lambda|^{2}=\overline{f(a)} f(a)=f\left(a^{*}\right) f(a)=f\left(a^{*} a\right)=f(e)=1 .
$$

This shows that $\sigma_{l}(a) \subset \Gamma$. Similarly, we can see that if $a$ is a normal element of $A$, then $\sigma_{l}(a)=\sigma_{r}(a)=\sigma(a)$. In particular, if $a$ is a unitary element of $A$, then $\sigma_{l}(a)=\sigma_{r}(a)=\sigma(a) \subset \Gamma$.

Let $T$ be an essentially isometric operator on $H$. Since $\pi(T)^{*} \pi(T)=\pi\left(I_{H}\right)$, it follows from what is showed above that $\sigma_{l e}(T)=\sigma_{l}(\pi(T)) \subset \Gamma$. Notice also that if $T$ is essentially unitary, then $\sigma_{l e}(T)=\sigma_{r e}(T)=\sigma_{e}(T) \subset \Gamma$.

The following result is probably known. Not being able to find a ready reference, we include a proof of it.

Proposition 2.3. (a) If $V$ is a nonunitary isometry on $H$, then

$$
\sigma_{l}(V)=\Gamma ; \sigma_{r}(V)=\sigma(V)=\bar{D} .
$$

(b) If $V$ is an arbitrary isometry on $H$, then

$$
\sigma_{l}(V)=\sigma_{r}(V) \cap \Gamma=\sigma(V) \cap \Gamma .
$$

Proof. (a) As we have seen above, $\sigma_{l}(V) \subset \Gamma$. On the other hand, we know that if $V$ is nonunitary isometry, then $\sigma(V)=\bar{D}$. It follows that $\Gamma=\partial \sigma(V) \subset \sigma_{l}(V)$.

Let $\lambda \in D$. Since $\lambda \in \sigma(V)$, from the relation

$$
\left\|\left(V-\lambda I_{H}\right) x\right\| \geq(1-|\lambda|)\|x\| \quad(x \in H)
$$

we deduce that the range of $V-\lambda I_{H}$ is closed and $\left(V-\lambda I_{H}\right) H \neq H$. Consequently, $V^{*} x=\bar{\lambda} x$ for some $x \in H \backslash\{0\}$. On the other hand, we know that for any $T \in B(H)$,

$$
\sigma_{r}(T)=\left\{\lambda \in \mathbb{C}: \inf \left\|\left(T^{*}-\bar{\lambda}\right) x\right\|=0,\|x\|=1\right\}
$$

[3, p.200]. It follows that $\lambda \in \sigma_{r}(V)$ and therefore, $D \subset \sigma_{r}(V)$. Since $\sigma_{r}(V)$ is closed, we have $\sigma_{r}(V)=\bar{D}$.

(b) follows from (a) and the fact that if $V$ is unitary, then $\sigma_{l}(V)=\sigma_{r}(V)=$ $\sigma(V)$.

As we have seen above if $V$ is a nonunitary isometry, then $\sigma(V)=\bar{D}$. It follows from the von Neumann inequality and the spectral theorem that for an arbitrary isometry $V$ on $H$,

$$
\|f(V)\|=\sup _{\xi \in \sigma(V) \cap \Gamma}|f(\xi)|, \forall f \in A(D) .
$$

Let $H_{0}$ be the linear space of all weakly null sequences $\left\{x_{n}\right\}$ in $H$. Let us define a semi-inner product on $H_{0}$ by

$$
\left\langle\left\{x_{n}\right\},\left\{y_{n}\right\}\right\rangle=\text { l.i.m. }_{n}\left\langle x_{n}, y_{n}\right\rangle,
$$


where l.i.m. is a Banach limit. Let

$$
E=\left\{\left\{x_{n}\right\} \in H_{0} \text { : l.i.m. }{ }_{\cdot n}\left\|x_{n}\right\|^{2}=0\right\} .
$$

Then, $H_{0} / E$ becomes a pre-Hilbert space with respect to the inner product defined by

$$
\left\langle\left\{x_{n}\right\}+E,\left\{y_{n}\right\}+E\right\rangle=\text { l.i.m.n }{ }_{n}\left\langle x_{n}, y_{n}\right\rangle .
$$

Let $\mathcal{H}$ be the completion of $H_{0} / E$ with respect to the induced norm given by

$$
\left\|\left\{x_{n}\right\}+E\right\|=\left(\text { l.i.m.n }_{n}\left\|x_{n}\right\|^{2}\right)^{\frac{1}{2}} .
$$

Then, $\mathcal{H}$ is a Hilbert space.

For a given $T \in B(H)$, define the operator $\mathcal{T}$ on $H_{0} / E$ by

$$
\mathcal{T}:\left\{x_{n}\right\}+E \mapsto\left\{T x_{n}\right\}+E .
$$

Then we have

$$
\begin{aligned}
\left\|\mathcal{T}\left(\left\{x_{n}\right\}+E\right)\right\| & =\left(\text { l.i.m.n } n\left\|T x_{n}\right\|^{2}\right)^{\frac{1}{2}} \\
& \leq\|T\|\left(\text { l.i.m.n }\left\|x_{n}\right\|^{2}\right)^{\frac{1}{2}} \\
& =\|T\|\left\|\left\{x_{n}\right\}+E\right\| .
\end{aligned}
$$

Since $H_{0} / E$ is dense in $\mathcal{H}$, the operator $\mathcal{T}$ can be extended to the whole $\mathcal{H}$ which we also denote by $\mathcal{T}$. Clearly, $\|\mathcal{T}\| \leq\|T\|$. The pair $(\mathcal{H}, \mathcal{T})$ (sometimes the operator $\mathcal{T}$ ) will be called the limit operator associated with $T$ (see also [11]).

Proposition 2.4. Let $T \in B(H)$ and let $(\mathcal{H}, \mathcal{T})$ be the limit operator associated with $T$. The following assertions hold:

(a) The mapping $T \mapsto \mathcal{T}$ is a contractive algebra $*$-homomorphism.

(b) $T$ is compact if and only if $\mathcal{T}=0$.

(c) $\sigma_{l}(\mathcal{T}) \subset \sigma_{l e}(T), \sigma_{r}(\mathcal{T}) \subset \sigma_{r e}(T)$, and $\sigma(\mathcal{T}) \subset \sigma_{e}(T)$.

(d) $T$ is an essentially isometric (resp. essentially unitary, essentially normal) operator if and only if $\mathcal{T}$ is an isometry (resp. unitary, normal).

(e) If $T$ is an essentially isometric operator and if $\sigma_{l e}(T) \neq \Gamma\left(\right.$ or $\left.\sigma_{r e}(T) \neq \bar{D}\right)$, then $T$ is essentially unitary.

Proof. The proof of (a) being very easy is omitted.

(b) It is obvious that if $T$ is compact, then $\mathcal{T}=0$. If $\mathcal{T}=0$, then for every weakly null sequence $\left\{x_{n}\right\}_{n \in \mathbb{N}}$ in $H$, we have l.i.m.n $\left\|T x_{n}\right\|^{2}=0$. Consequently, there is a subsequence $\left\{x_{n_{k}}\right\}_{k \in \mathbb{N}}$ of $\left\{x_{n}\right\}_{n \in \mathbb{N}}$ such that

$$
\varlimsup_{n \rightarrow \infty}\left\|T x_{n}\right\|^{2}=\lim _{k \rightarrow \infty}\left\|T x_{n_{k}}\right\|^{2}=\text { l.i.m. } ._{k}\left\|T x_{n_{k}}\right\|^{2}=0 .
$$

It follows that $\lim _{n \rightarrow \infty}\left\|T x_{n}\right\|=0$ and therefore $T$ is compact.

(c) If $\lambda \notin \sigma_{l e}(T)$, then $\lambda I_{H}-T$ is a left Fredholm operator. So, there exists $S \in B(H)$ such that $S\left(\lambda I_{H}-T\right)-I_{H} \in K(H)$. It follows from (a) and (b) that $\mathcal{S}\left(\lambda I_{\mathcal{H}}-\mathcal{T}\right)=I_{\mathcal{H}}$, where $\mathcal{S}$ is the limit operator associated with $S$. This shows that $\lambda \notin \sigma_{l}(\mathcal{T})$. The proofs of the second and third parts of (c) are similar.

(d) is an immediate consequence of (a) and (b).

(e) Assume that $T$ is an essentially isometric operator and $\sigma_{l e}(T) \neq \Gamma$ (or $\left.\sigma_{r e}(T) \neq \bar{D}\right)$. By $(\mathrm{c})$ we have $\sigma_{l}(\mathcal{T}) \neq \Gamma\left(\right.$ or $\left.\sigma_{r}(\mathcal{T}) \neq \bar{D}\right)$. Since $\mathcal{T}$ is an isometry, 
it follows from Proposition 2.3 that $\mathcal{T}$ is unitary. Consequently, both $I-T^{*} T$ and $I-T T^{*}$ are compact.

We are now able to prove Theorem 2.2.

Proof of Theorem 2.2. Assume that the Gelfand transform of $S \in A_{T}$ vanishes on $\sigma_{l e}(T)$ (or on $\sigma_{r e}(T) \cap \Gamma$ ). Since $S \in A_{T}$, there exists a sequence of polynomials $\left\{P_{n}\right\}$ such that

$$
\lim _{n \rightarrow \infty}\left\|P_{n}(T)-S\right\|=0 .
$$

Let $\mathcal{T}$ and $\mathcal{S}$ be the limit operators associated with $T$ and $S$, respectively. In view of Proposition 2.4 (a), we have

$$
\lim _{n \rightarrow \infty}\left\|P_{n}(\mathcal{T})-\mathcal{S}\right\|=0
$$

On the other hand, for every $\xi \in \sigma_{l e}(T)\left(\xi \in \sigma_{r e}(T) \cap \Gamma\right)$ there exists a multiplicative functional $\phi_{\xi}$ on $A_{T}$ such that $\phi_{\xi}(T)=\xi$. Consequently, we have

$$
\begin{aligned}
\left|P_{n}(\xi)\right| & =\left|P_{n}(\xi)-\widehat{S}(\xi)\right| \\
& =\left|\phi_{\xi}\left(P_{n}(T)-S\right)\right| \\
& \leq\left\|P_{n}(T)-S\right\| .
\end{aligned}
$$

From this we deduce that $\lim _{n \rightarrow \infty} P_{n}(\xi)=0$ uniformly on $\sigma_{l e}(T)\left(\right.$ on $\left.\sigma_{r e}(T) \cap \Gamma\right)$. Further, it follows from Proposition 2.4 (d), (c), and Proposition 2.3 that $\mathcal{T}$ is an isometry and

$$
\begin{aligned}
\sigma(\mathcal{T}) \cap \Gamma & =\sigma_{l}(\mathcal{T}) \subset \sigma_{l e}(T) \\
(\sigma(\mathcal{T}) \cap \Gamma & \left.=\sigma_{r}(\mathcal{T}) \cap \Gamma \subset \sigma_{r e}(T) \cap \Gamma\right) .
\end{aligned}
$$

Consequently, $\lim _{n \rightarrow \infty} P_{n}(\xi)=0$ uniformly on $\sigma(\mathcal{T}) \cap \Gamma$. Now, taking into account the identity $(2.1)$, we obtain

$$
\lim _{n \rightarrow \infty}\left\|P_{n}(\mathcal{T})\right\|=0 .
$$

Hence, $\mathcal{S}=0$. By Proposition 2.4 (b), $S$ is compact.

The following proposition is an improvement of [6, Proposition 2.5] and shows that the condition " $T$ is essentially isometric" is necessary in the Theorem 2.2.

Proposition 2.5. (a) Let $T$ be a contraction on $H$ and let $K$ be a closed subset of $\Gamma$ of Lebesgue measure zero. Assume that $f(T)$ is compact for every $f \in A(D)$ vanishing on $K$. Then, $T$ is essentially unitary and $\sigma_{e}(T) \subset K$.

(b) Let $T$ be an essentially unitary, but nonunitary contraction such that $\sigma_{e}(T)$ is of Lebesgue measure zero. Then, there exists $f \in A(D)$ such that $f(T)$ is a nonzero compact operator.

Proof. (a) Let $\pi: B(H) \rightarrow B(H) / K(H)$ be the canonical map. By RudinCarleson Theorem [1, Theorem VIII.7.4], there exists $f \in A(D)$ such that $f(\xi)=$ $\bar{\xi}$ for all $\xi \in K$ and $\|f\|_{\infty}=1$. Since the function $z f(z)-1$ vanishes on $K$, the operator $T f(T)-I_{H}$ is compact. Consequently, we have $\pi(T) \pi(f(T))=\pi\left(I_{H}\right)$. This imply that $\pi(T)$ is invertible and

$$
\left\|\pi(T)^{-1}\right\|=\|\pi(f(T))\| \leq\|f(T)\| \leq\|f\|_{\infty} \leq 1 .
$$


Since $\|\pi(T)\| \leq 1$, we have $\|\pi(T)\|=\left\|\pi(T)^{-1}\right\|=1$. This shows that $\pi(T)$ is unitary and therefore $T$ is essentially unitary.

We have $\sigma_{e}(T) \subset \Gamma$. Let us show that $\sigma_{e}(T) \subset K$. Let $\xi_{0} \in \Gamma \backslash K$. By RudinCarleson Theorem, there exists $f \in A(D)$ such that $f(\xi)=\left(\xi_{0}-\xi\right)^{-1}$ on $K$. Since the function $\left(\xi_{0}-z\right) f(z)-1$ vanishes on $K$, the operator $\left(\xi_{0} I_{H}-T\right) f(T)-$ $I_{H}$ is compact. This shows that $\xi_{0} \notin \sigma_{e}(T)$.

(b) By Theorem 2.2, it suffices to show that there exists $f \in A(D)$ such that $f$ vanishes on $\sigma_{e}(T)$, but $f(T) \neq 0$. Assume on the contrary that $f(T)=0$ for every $f \in A(D)$ vanishing on $\sigma_{e}(T)$. By Rudin-Carleson Theorem, there exists $f \in A(D)$ such that $f(\xi)=\bar{\xi}$ for all $\xi \in K$ and $\|f\|_{\infty}=1$. Since the function $z f(z)-1$ vanishes on $K$, we have $T f(T)=I_{H}$. Consequently, $T$ is invertible and

$$
\left\|T^{-1}\right\|=\|f(T)\| \leq\|f\|_{\infty} \leq 1 .
$$

Thus we have $\|T\|=\left\|T^{-1}\right\|=1$. This shows that $T$ is unitary. This is a contradiction.

Recall [15, III.1] that the spectrum $\Sigma(\varphi)$ of an inner function $\varphi$ is defined by

$$
\Sigma(\varphi)=\overline{\varphi^{-1}(0)} \cup \operatorname{supp} \mu
$$

where $\mu$ is the singular measure associated to the singular part of $\varphi$. As is known [13, Proposition III.4.4] if $T$ is a $C_{0}$-contraction, then there exists a minimal inner function $m_{T}$ that annihilates $T$, i.e., $m_{T}(T)=0$ and we have $\sigma(T)=\Sigma\left(m_{T}\right)$. Now, it follows from Proposition 2.4 (e) that if $T$ is an essentially isometric $C_{0}$-contraction, then it is essentially unitary. In fact, $T$ is a compact perturbation of a unitary operator $[6,17]$.

Corollary 2.6. If $T$ is an essentially isometric $C_{0}$-contraction on $H$, then there exist a nonzero $T$-invariant subspace $E$ and $f \in A(D)$ such that $f\left(\left.T\right|_{E}\right)$ is a nonzero compact operator.

Proof. Let $m_{T}$ be the minimal inner function that annihilates $T$. Then, there exists an inner function $\theta$ such that $\theta$ divides $m_{T}$ and $\Sigma(\theta) \cap \Gamma$ is of Lebesgue measure zero $[6,16]$. Let

$$
\psi:=\frac{m_{T}}{\theta} ; E:=\overline{\psi(T) H}
$$

The minimality of $m_{T}$ implies that $E \neq\{0\}$ and $\left.T\right|_{E}$ is a $C_{0}$-contraction with $m_{\left.T\right|_{E}}=\theta$. Moreover, the operator

$$
I_{E}-\left(\left.T\right|_{E}\right)^{*}\left(\left.T\right|_{E}\right)=\left.P_{E}\left(I_{H}-T^{*} T\right)\right|_{E}
$$

is compact, where $P_{E}$ is the orthogonal projection from $H$ onto $E$. As we already noted above, essentially isometric $C_{0}$-contractions are essentially unitary. Thus, $\left.T\right|_{E}$ is an essentially unitary (but nonunitary) contraction and $\sigma\left(\left.T\right|_{E}\right) \cap \Gamma$ $(=\Sigma(\theta) \cap \Gamma)$ is of Lebesgue measure zero. By Proposition 2.5 (b), there exists $f \in A(D)$ such that $f\left(\left.T\right|_{E}\right)$ is a nonzero compact operator.

We already noted in the introduction that if $T$ is an essentially unitary $C_{0}$-contraction, then $H^{\infty}(T)$ contains a nonzero compact operator [16]. Notice that this result can be derived form the preceding corollary as follows. Let $\psi, E$, and $f$ be 
as in the proof of Corollary 2.6. Then, $f\left(\left.T\right|_{E}\right)$ is a nonzero compact operator. Now, from the identity $f(T) \psi(T)=f\left(\left.T\right|_{E}\right) \psi(T)$ we deduce that $f(T) \psi(T)$ is a nonzero compact operator, which is contained in $H^{\infty}(T)$.

If $T$ is a contraction on $H$, then there exists a canonical decomposition of $H$ into two $T$-invariant subspaces $H=H_{0} \oplus H_{u}$ such that $T_{0}:=\left.T\right|_{H_{0}}$ is a c.n.u. contraction and $T_{u}:=\left.T\right|_{H_{u}}$ is unitary [13, I.3.2]. It can be seen that $\sigma\left(T_{u}\right) \subset \sigma(T) \cap \Gamma$. For a nonempty closed subset $S$ of $\Gamma$, by $H_{S}^{\infty}$ we denote the set of all those $f$ in $H^{\infty}$ that have a continuous extension $\bar{f}$ to $D \cup S$. If $f \in H_{\sigma(T) \cap \Gamma}^{\infty}$ with continuous extension $\bar{f}$ to $D \cup(\sigma(T) \cap \Gamma)$, then we can define $f(T) \in B(H)$, by

$$
f(T)=f\left(T_{0}\right) \oplus \bar{f}\left(T_{u}\right),
$$

where $f\left(T_{0}\right)$ is given by the Nagy-Foias functional calculus and

$$
\bar{f}\left(T_{u}\right)=\left(\left.\bar{f}\right|_{\sigma(T) \cap \Gamma}\right)\left(T_{u}\right)
$$

is defined by the usual functional calculus for continuous functions of a unitary operator (see also [5]). Notice that

$$
\|f(T)\| \leq\|f\|_{\infty}, \forall f \in H_{\sigma(T) \cap \Gamma}^{\infty} .
$$

Now, let $f \in H_{\sigma(T) \cap \Gamma}^{\infty}$ with continuous extension $\bar{f}$ to $D \cup(\sigma(T) \cap \Gamma)$. By the Gamelin-Garnett Theorem [4], there exists a sequence $\left\{f_{n}\right\}$ in $H^{\infty}$ such that

$$
\lim _{n \rightarrow \infty}\left\|f_{n}-f\right\|_{\infty}=0
$$

and each $f_{n}$ has an analytic extension $g_{n}$ to an open set $O_{n}$ containing $D \cup$ $(\sigma(T) \cap \Gamma)$. Then, $g_{n}(T)$ can be defined by the Riesz-Dunford functional calculus and coincides with $f_{n}(T)$, where $f_{n}(T)$ is defined as above. Notice also that $g_{n}(T)$ is in $A_{T}$. Consequently, we have

$$
\left\|g_{n}(T)-f(T)\right\|=\left\|f_{n}(T)-f(T)\right\| \leq\left\|f_{n}-f\right\|_{\infty} \rightarrow 0(n \rightarrow \infty) .
$$

It follows that $f(T) \in A_{T}$.

The next corollary is now an immediate consequence of Theorem 2.2.

Corollary 2.7. Let $T$ be an essentially isometric contraction and let $f \in H_{\sigma(T) \cap \Gamma}^{\infty}$ with continuous extension $\bar{f}$ to $D \cup(\sigma(T) \cap \Gamma)$. If $\bar{f}(\xi)=0$ on $\sigma(T) \cap \Gamma$, then $f(T)$ is compact.

Corollary 2.8. Let $T$ be an essentially unitary c.n.u. contraction such that $\sigma(T) \cap \Gamma$ is of Lebesgue measure zero. Then,

$$
\sigma_{e}(T)=\sigma(T) \cap \Gamma .
$$

Proof. Assume on the contrary that there exists $\xi_{0} \in \sigma(T) \cap \Gamma$, but $\xi_{0} \notin \sigma_{e}(T)$. Then, there exists a continuous function $f_{0}$ on $\sigma(T) \cap \Gamma$ such that $f_{0}\left(\xi_{0}\right) \neq 0$ and $f_{0}(\xi)=0$ for all $\xi \in \sigma_{e}(T)$. Let $f \in A(D)$ be the Rudin-Carleson extension of $f_{0}$. By Theorem 2.2, $f(T)$ is compact. On the other hand, it follows from Proposition 2.1 that $f$ vanishes on $\sigma(T) \cap \Gamma$. This contradicts $f_{0}\left(\xi_{0}\right) \neq 0$. 
Recall that a contraction $T$ on $H$ is said to be of class $C_{00}$ if $T^{n} x \rightarrow 0$ and $T^{* n} x \rightarrow 0$ for every $x \in H$.

Assume that the contraction $T$ is of class $C_{00}$. Moreover, assume that

$$
\operatorname{dim}\left(I-T T^{*}\right) H=\operatorname{dim}\left(I-T^{*} T\right) H=1
$$

(consequently, $T$ is essentially unitary). According to the well-known model theorem of Nagy-Foias [13, 15], $T$ is unitary equivalent to its model operator $M_{\varphi}=\left.P_{\varphi} S\right|_{K_{\varphi}}$ acting on the model space $K_{\varphi}:=H^{2} \ominus \varphi H^{2}$, where $\varphi$ is an inner function, $S f=z f$ is the shift operator on the Hardy space $H^{2}$, and $P_{\varphi}$ is the orthogonal projection from $H^{2}$ onto $K_{\varphi}$. It follows that for every $f \in H^{\infty}$, the operator $f(T)$ is unitary equivalent to

$$
f\left(M_{\varphi}\right):=\left.P_{\varphi} f(S)\right|_{K_{\varphi}} .
$$

As is known [15, p.235], $\{T\}^{\prime}=\left\{f(T): f \in H^{\infty}\right\}$. By Hartman-Sarason theorem [15, p.235], $f(T)\left(f \in H^{\infty}\right)$ is compact if and only if $\lim _{n \rightarrow \infty}\left\|T^{n} f(T)\right\|=0$.

We have the following

Theorem 2.9. If $T$ is an essentially isometric c.n.u. contraction, then $S \in\{T\}^{\prime}$ is compact if and only if

$$
\lim _{n \rightarrow \infty}\left\|T^{n} S\right\|=0
$$

Proof. Assume that $S \in\{T\}^{\prime}$ is compact. Since $T$ is a c.n.u. contraction, $T^{n} \rightarrow 0$ in the weak operator topology. Consequently, for every $x \in H$ we can write

$$
\lim _{n \rightarrow \infty}\left\|T^{n} S x\right\|=\lim _{n \rightarrow \infty}\left\|S T^{n} x\right\|=0 .
$$

As in the proof of Proposition 2.1, we have

$$
\lim _{n \rightarrow \infty}\left\|T^{n} S\right\|=0 .
$$

Let $\mathcal{T}$ and $\mathcal{S}$ be the limit operators associated with $T$ and $S$, respectively. By Proposition 2.4 (a),

$$
\left\|\mathcal{T}^{n} \mathcal{S}\right\| \leq\left\|T^{n} S\right\|, \forall n \in \mathbb{N} .
$$

Since $\mathcal{T}$ is an isometry, we have

$$
\|\mathcal{S}\| \leq \lim _{n \rightarrow \infty}\left\|T^{n} S\right\|=0
$$

so that $\mathcal{S}=0$. By Proposition 2.4 (b), $S$ is compact.

Note that the preceding theorem contains the main results of [6].

In the proof of the following proposition we use the dilation arguments of Nagy-Foias (see, [13, p.140] and [17, Theorem 3.3]).

Proposition 2.10. Let $T$ be a c.n.u. contraction on $H$. Assume that there exists a nonzero function $f \in H^{\infty}$ such that $f(T)$ is compact. Then for every $S \in K(H)$, we have

$$
\lim _{n \rightarrow \infty}\left\|T^{n} S\right\|=\lim _{n \rightarrow \infty}\left\|S T^{n}\right\|=0 .
$$


Proof. Assume that $f(T)$ is compact for some nonzero $f \in H^{\infty}$. Since $T$ is a c.n.u. contraction, $T^{n} \rightarrow 0$ in the weak operator topology and therefore,

$$
\lim _{n \rightarrow \infty}\left\|T^{n} f(T) x\right\|=0, \forall x \in H .
$$

Let $f=f_{i} f_{e}$ be the canonical inner-outer factorization of $f$, where $f_{i}$ is inner and $f_{e}$ is outer function. Since $f_{e}(T)$ has dense range [13, Proposition III.3.1], we have

$$
\lim _{n \rightarrow \infty}\left\|T^{n} f_{i}(T) x\right\|=0, \forall x \in H .
$$

If $U$ is the minimal unitary dilation of $T$, then

$$
\lim _{n \rightarrow \infty} U^{-n} T^{n} x=P x
$$

where $P$ is the orthogonal projection onto the residual part of the dilation space [13, Proposition II.3.1]. It follows that

$$
\lim _{n \rightarrow \infty}\left\|T^{n} x\right\|=\|P x\| \quad(x \in H) .
$$

Let us show that $P x=0$. We can write

$$
U^{-m} P T^{m} x=\lim _{n \rightarrow \infty} U^{-m-n} T^{m+n} x=P x,
$$

which implies

$$
P T^{m} x=U^{m} P x \quad(m \in \mathbb{N}) .
$$

Consequently, we have

$$
P T^{n} f_{i}(T) x=U^{n} f_{i}(U) P x(n \in \mathbb{N}) .
$$

Since $f_{i}(U)$ is unitary, we can write

$$
\begin{aligned}
\|P x\| & =\left\|U^{n} f_{i}(U) P x\right\| \\
& =\left\|P T^{n} f_{i}(T) x\right\| \\
& \leq\left\|T^{n} f_{i}(T) x\right\| \rightarrow 0(n \rightarrow \infty) .
\end{aligned}
$$

Hence we have $\lim _{n \rightarrow \infty}\left\|T^{n} x\right\|=0$ which implies that

$$
\lim _{n \rightarrow \infty}\left\|T^{n} S x\right\|=0 \text { for every } S \in B(H) \text { and } x \in H .
$$

As in the proof of Proposition 2.1 we can see that if $S \in K(H)$, then

$$
\lim _{n \rightarrow \infty}\left\|T^{n} S\right\|=0 .
$$

Taking into account the fact that $f(T)^{*}=\widetilde{f}\left(T^{*}\right)$, where $\widetilde{f}(z)=\overline{f(\bar{z})}$, we can apply the above result to $T^{*}$ to obtain

$$
\lim _{n \rightarrow \infty}\left\|T^{* n} S^{*} x\right\|=0 \text { for every } S \in B(H) \text { and } x \in H .
$$

It follows that if $S \in K(H)$, then $\lim _{n \rightarrow \infty}\left\|S T^{n}\right\|=0$.

The following result is of independent interest (for related results see [10]). 
Proposition 2.11. Let $T$ be an essentially unitary c.n.u. contraction on $H$ such that $\sigma_{e}(T)$ is of Lebesgue measure zero. For every $S \in A_{T}$, we have

$$
\operatorname{dist}\left(S, A_{T} \cap K(H)\right)=\sup _{\xi \in \sigma_{e}(T)}|\widehat{S}(\xi)| .
$$

Proof. Let $S \in A_{T}, K \in A_{T} \cap K(H)$, and $\xi \in \sigma_{e}(T)$ be given. There exists a multiplicative functional $\phi_{\xi}$ on $A_{T}$ such that $\phi_{\xi}(T)=\xi$. Consequently, we have

$$
\begin{aligned}
|\widehat{S}(\xi)| & =\left|\phi_{\xi}\left(T^{n} S\right)\right| \leq\left\|T^{n} S\right\| \\
& \leq\left\|T^{n} S-T^{n} K\right\|+\left\|T^{n} K\right\| \\
& \leq\|S-K\|+\left\|T^{n} K\right\| .
\end{aligned}
$$

Since $T^{n} \rightarrow 0$ in the weak operator topology, as in the proof of Proposition 2.1 we have

$$
\lim _{n \rightarrow \infty}\left\|T^{n} K\right\|=0 .
$$

Letting $n \rightarrow \infty$ in the preceding inequality, we obtain $|\widehat{S}(\xi)| \leq\|S-K\|$. It follows that

$$
\sup _{\xi \in \sigma_{e}(T)}|\widehat{S}(\xi)| \leq \operatorname{dist}\left(S, A_{T} \cap K(H)\right) .
$$

To prove the opposite inequality, let $\varepsilon>0$ be given. Then there exists $f \in$ $A(D)$ such that $\|S-f(T)\| \leq \varepsilon$. It follows that

$$
\sup _{\xi \in \sigma_{e}(T)}|f(\xi)| \leq \sup _{\xi \in \sigma_{e}(T)}|\widehat{S}(\xi)|+\varepsilon .
$$

By Rudin-Carleson Theorem, there exists $g \in A(D)$ such that $g(\xi)=f(\xi)$ on $\sigma_{e}(T)$ and

$$
\|g\|_{\infty}=\sup _{\xi \in \sigma_{e}(T)}|f(\xi)| .
$$

Since $g-f$ vanishes on $\sigma_{e}(T)$, by Theorem 2.2, $g(T)-f(T)$ is compact. Hence, we can write

$$
\begin{aligned}
\operatorname{dist}\left(S, A_{T} \cap K(H)\right) & \leq\|S+g(T)-f(T)\| \\
& \leq\|g(T)\|+\varepsilon \\
& \leq\|g\|_{\infty}+\varepsilon \\
& =\sup _{\xi \in \sigma_{e}(T)}|f(\xi)|+\varepsilon \\
& \leq \sup _{\xi \in \sigma_{e}(T)}|\widehat{S}(\xi)|+2 \varepsilon .
\end{aligned}
$$

Since $\varepsilon$ was arbitrary, we obtain that

$$
\operatorname{dist}\left(S, A_{T} \cap K(H)\right) \leq \sup _{\xi \in \sigma_{e}(T)}|\widehat{S}(\xi)|
$$

Next, we characterize the compactness via the ergodic conditions. The following lemma was proved in [8, Lemma 2.4]. 
Lemma 2.12. Let $V$ be an isometry on $H$ and let $S \in\{V\}^{\prime}$. If

$$
\underline{\lim _{n \rightarrow \infty}} \frac{1}{n}\left\|\sum_{k=1}^{n} \bar{\xi}^{k} V^{k} S\right\|=0
$$

holds for every $\xi \in \sigma(V) \cap \Gamma$, then $S=0$.

As an application, we have the following

Theorem 2.13. Let $T$ be an essentially isometric operator and let $S \in\{T\}^{\prime}$. If

$$
\varliminf_{n \rightarrow \infty} \frac{1}{n}\left\|\sum_{k=1}^{n} \bar{\xi}^{k} T^{k} S\right\|=0
$$

holds for every $\xi \in \sigma_{l e}(T)$ (or $\left.\xi \in \sigma_{\text {re }}(T) \cap \Gamma\right)$, then $S$ is compact.

Proof. Let $\mathcal{T}$ and $\mathcal{S}$ be the limit operators associated with $T$ and $S$, respectively. From Proposition 2.4 (d), (c), and Proposition 2.3 we deduce that $\mathcal{T}$ is an isometry and

$$
\sigma(\mathcal{T}) \cap \Gamma \subset \sigma_{l e}(T) \quad\left(\text { or } \sigma(\mathcal{T}) \cap \Gamma \subset \sigma_{r e}(T) \cap \Gamma\right)
$$

Furthermore, $\mathcal{S} \in\{\mathcal{T}\}^{\prime}$. Now, it follows from Proposition 2.4 (a) that

$$
\varliminf_{n \rightarrow \infty} \frac{1}{n}\left\|\sum_{k=1}^{n} \bar{\xi}^{k} \mathcal{T}^{k} \mathcal{S}\right\|=0
$$

holds for every $\xi \in \sigma(\mathcal{T}) \cap \Gamma$. By the preceding lemma, $\mathcal{S}=0$. Consequently, by Proposition 2.4 (b), $S$ is compact.

\section{Essentially nORMal operators}

Let $T$ be an essentially normal operator, that is, $T T^{*}-T^{*} T$ is compact. Since $\pi(T)$ is a normal element of the Calkin algebra, we have $\sigma_{l e}(T)=\sigma_{r e}(T)=$ $\sigma_{e}(T)$. It will be useful to note that if $\operatorname{ind}\left(T-\lambda I_{H}\right)=0$ for every $\lambda \in \mathbb{C} \backslash \sigma_{e}(T)$, then $T$ is a compact perturbation of a normal operator [2].

In this section, for the compactness of $S$ from the commutant of $T$, some necessary and sufficient conditions are found on $S$. The compactness of $S \in\{T\}^{\prime}$ via the ergodic conditions is also characterized.

The first main result of this section is the following

Theorem 3.1. Let $T$ be an essentially normal operator. If the Gelfand transform of $S \in A_{T}$ vanishes on $\sigma_{e}(T)$, then $S$ is compact.

Proof. Assume that the Gelfand transform of $S \in A_{T}$ vanishes on $\sigma_{e}(T)$. Since $S \in A_{T}$, there exists a sequence of polynomials $\left\{P_{n}\right\}$ such that

$$
\lim _{n \rightarrow \infty}\left\|P_{n}(T)-S\right\|=0 .
$$

Let $\mathcal{T}$ and $\mathcal{S}$ be the limit operators associated with $T$ and $S$, respectively. In view of Proposition 2.4 (a), we have

$$
\lim _{n \rightarrow \infty}\left\|P_{n}(\mathcal{T})-\mathcal{S}\right\|=0 .
$$


Further, for every $\lambda \in \sigma_{e}(T)$, there exists a multiplicative functional $\phi_{\lambda}$ on $A_{T}$ such that $\phi_{\lambda}(T)=\lambda$. Consequently, we can write

$$
\begin{aligned}
\left|P_{n}(\lambda)\right| & =\left|P_{n}(\lambda)-\widehat{S}(\lambda)\right| \\
& =\left|\phi_{\lambda}\left(P_{n}(T)-S\right)\right| \\
& \leq\left\|P_{n}(T)-S\right\| .
\end{aligned}
$$

From this, we deduce that $\lim _{n \rightarrow \infty} P_{n}(\lambda)=0$ uniformly on $\sigma_{e}(T)$. On the other hand, it follows from Proposition 2.4 (c) and (d) that $\mathcal{T}$ is a normal operator and $\sigma(\mathcal{T}) \subset \sigma_{e}(T)$. Consequently, $\lim _{n \rightarrow \infty} P_{n}(\lambda)=0$ uniformly on $\sigma(\mathcal{T})$. It follows that

$$
\lim _{n \rightarrow \infty}\left\|P_{n}(\mathcal{T})\right\|=0 .
$$

Thus we obtain $\mathcal{S}=0$. By Proposition 2.4 (b), $S$ is compact.

Corollary 3.2. If $T$ is an essentially normal operator on $H$, then the following assertions hold:

(a) If $\sigma_{e}(T)=\left\{\lambda_{1}, \cdots, \lambda_{n}\right\}$, then $\left(T-\lambda_{1} I_{H}\right) \cdots\left(T-\lambda_{n} I_{H}\right)$ is compact.

(b) The radical Rad $\left(A_{T}\right)$ of the algebra $A_{T}$ consists of Volterra operators.

Proof. (a) The Gelfand transform of $S:=\left(T-\lambda_{1} I_{H}\right) \cdots\left(T-\lambda_{n} I_{H}\right)$ vanishes on $\left\{\lambda_{1}, \cdots, \lambda_{n}\right\}$. By Theorem 3.1, $S$ is compact.

(b) If $R \in \operatorname{Rad}\left(A_{T}\right)$, then $\widehat{R}$ vanishes on $\sigma(T)$. Since $\sigma_{e}(T) \subset \sigma(T)$, it follows that $\widehat{R}$ vanishes on $\sigma_{e}(T)$. By Theorem 3.1, $S$ is compact.

Next, we will prove the following

Proposition 3.3. Let $T$ be an essentially normal operator such that

$$
\sigma_{e}(T) \subset\{\lambda \in \mathbb{C}:|\lambda| \geq 1\}
$$

and let $S \in B(H)$. If

$$
\varliminf_{n \rightarrow \infty}\left\|T^{n} S\right\|=0,
$$

then $S$ is compact.

Proof. Let $\mathcal{T}$ and $\mathcal{S}$ be the limit operators associated with $T$ and $S$, respectively. It follows from Proposition 2.4 (d) and (c) that $\mathcal{T}$ is normal and

$$
\sigma(\mathcal{T}) \subset \sigma_{e}(T) \subset\{\lambda \in \mathbb{C}:|\lambda| \geq 1\} .
$$

On the other hand, we have

$$
\|\mathcal{S}\| \leq\left\|\mathcal{T}^{-n}\right\|\left\|\mathcal{T}^{n} \mathcal{S}\right\|=\sup _{\lambda \in \sigma(\mathcal{T})}|\lambda|^{-n}\left\|\mathcal{T}^{n} \mathcal{S}\right\| \leq\left\|T^{n} S\right\| .
$$

This clearly implies that $\mathcal{S}=0$. By Proposition 2.4 (b), $S$ is compact.

Below, we characterize the compactness via the ergodic conditions.

Theorem 3.4. Let $T$ be an essentially normal Fredholm operator and let $S \in$ $\{T\}^{\prime}$. If

$$
\varliminf_{n \rightarrow \infty} \frac{1}{n}\left\|\sum_{k=1}^{n} \lambda^{-k} T^{k} S\right\|=0
$$


holds for every $\lambda \in \sigma_{e}(T)$, then $S$ is compact.

We shall need the following

Lemma 3.5. Let $N$ be an invertible normal operator and let $S \in\{N\}^{\prime}$. If

$$
\varliminf_{n \rightarrow \infty} \frac{1}{n}\left\|\sum_{k=1}^{n} \lambda^{-k} N^{k} S\right\|=0
$$

holds for every $\lambda \in \sigma(N)$, then $S=0$.

Proof. It suffices to show that $S S^{*}=0$. By Fuglede-Putnam theorem, $S N^{*}=$ $N^{*} S$ which implies $N S^{*}=S^{*} N$. Consequently, we can write

$$
N\left(S S^{*}\right)=(N S) S^{*}=(S N) S^{*}=S\left(N S^{*}\right)=S\left(S^{*} N\right)=\left(S S^{*}\right) N .
$$

So, $N$ commutes with $S S^{*}$. Let $A$ be the unital $C^{*}$-algebra generated by $N$ and $S S^{*}$. Then, $A$ is commutative. Denote by $\Sigma$ the Gelfand spectrum of $A$. Since the algebra $A$ is isomprphic to $C(\Sigma)$, it suffices to show that $\phi\left(S S^{*}\right)=0$ for all $\phi \in \Sigma$. Notice also that $A$ is a full subalgebra of $B(H)$ and therefore, $\sigma(N)=\{\phi(N): \phi \in \Sigma\}$. If $\phi \in \Sigma$ and if $\lambda:=\phi(N)$, then we have

$$
\begin{aligned}
\left|\phi\left(S S^{*}\right)\right| & =\frac{1}{n}\left|\sum_{k=1}^{n} \lambda^{-k} \phi(N)^{k} \phi\left(S S^{*}\right)\right| \\
& =\frac{1}{n}\left|\left\langle\phi, \sum_{k=1}^{n} \lambda^{-k} N^{k} S S^{*}\right\rangle\right| \\
& \leq \frac{1}{n}\left\|\sum_{k=1}^{n} \lambda^{-k} N^{k} S\right\|\left\|S^{*}\right\| .
\end{aligned}
$$

Taking lower limit as $n \rightarrow \infty$, we get $\phi\left(S S^{*}\right)=0$.

Proof of Theorem 3.4. Let $\mathcal{T}$ and $\mathcal{S}$ be the limit operators associated with $T$ and $S$, respectively. In view of Proposition 2.4 (c) and (d), $\mathcal{T}$ is an invertible normal operator and $\sigma(\mathcal{T}) \subset \sigma_{e}(T)$. Furthermore, $\mathcal{S} \in\{\mathcal{T}\}^{\prime}$. Now, it follows from Proposition 2.4 (a) that

$$
\varliminf_{n \rightarrow \infty} \frac{1}{n}\left\|\sum_{k=1}^{n} \lambda^{-k} \mathcal{T}^{k} \mathcal{S}\right\|=0
$$

holds for every $\lambda \in \sigma(\mathcal{T})$. By the preceding lemma, $\mathcal{S}=0$. Consequently, by Proposition 2.4 (b), $S$ is compact.

Acknowledgement. The author would like to thank the referee for many valuable and useful comments and suggestions which have improved this paper.

\section{REFERENCES}

1. B. Beauzamy, Introduction to Operator Theory and Invariant Subspaces, North-Holland Mathematical Library, 42, North Holland, Amsterdam, 1988. 
2. L.G. Brown, R.G. Douglas and P.A. Filmore, Unitary equivalence modulo the compact operators and extensions of $C^{*}$-algebras, Proceedings of a Conference on Operator Theory, Lecture Notes in Math., Springer, Berlin, 345 (1973), 58-128.

3. J.B. Conway, A Course in Functional Analysis, Graduate Texts in Mathematics, 96, Springer-Verlag, New York, 1985.

4. T. Gamelin and J. Garnett, Uniform approximation to bounded analytic functions,. Rev. Un. Mat. Argentina 25 (1970), 87-94.

5. I.B. Jung, E. Ko and C. Pearcy, A note on the spectral mapping theorem, Kyungpook Math. J. 47 (2007), 77-79.

6. K. Kellay and M. Zarrabi, Compact operators that commute with a contraction, Integral equations Operator Theory 65 (2009), 543-550.

7. R. Larsen, Banach Algebras, Pure and Applied Mathematics, 24, Marcel Dekker Inc., New York, 1973.

8. Z. Léka, A Katznelson-Tzafriri type theorem in Hilbert spaces, Proc. Amer. Math. Soc. 137 (2009), 3763-3768.

9. P. Muhly, Compact operators in the commutant of a contraction, J. Funct. Anal. 8 (1971), $197-224$.

10. H.S. Mustafayev, Asymptotic behavior of polynomially bounded operators, C. R. Acad. Sci. Paris, Ser.I 348 (2010), 517-520.

11. H.S. Mustafayev, The essential spectrum of the essentially isometric operator, Canad. Math. Bull. 57 (2014), 145-158.

12. B. Sz.-Nagy, On a property of operators of class $C_{0}$, Acta Sci. Math. (Szeged) 36 (1974), 219-220.

13. B. Sz.-Nagy and C. Foias, Harmonic Analysis of Operators on Hilbert Space(Russian), Mir, Moscow, 1970.

14. M.A. Naimark, Normed Rings (Russian), Nauka, Moscow, 1968.

15. N.K. Nikolski, Lectures on the shift operator (Russian), Nauka, Moscow, 1980.

16. E.A. Nordgren, Compact operators in the algebra generated by essentially unitary $C_{0}$ operators, Proc. Amer. Math. Soc. 51 (1975), 159-162.

17. P. Vitse, Smooth operators in the commutant of a contraction, Studia Math. 155 (2003), 241-263.

${ }^{1}$ Yuzuncu Yil University, Faculty of Sciences, Department of Mathematics, 65080, VAN, TURKEY.

E-mail address: hsmustafayev@yahoo.com

${ }^{2}$ Azerbaijan Pedagogical University, Department of Mathematics, Baku, AzerBAIJAN.

E-mail address: fbhuseynov@yahoo.com 